\title{
Effect of exclusive enteral nutrition on colonic bacterial activity in paediatric Crohn's disease
}

\author{
Konstantinos Gerasimidis ${ }^{1}$, Paraic McGrogan ${ }^{2}$, Vikki Garrick ${ }^{2}$, Kamal Hassan ${ }^{2}$ \\ and Christine Ann Edwards ${ }^{1}$ \\ ${ }^{1}$ Human Nutrition Section, Developmental Medicine, University of Glasgow, Glasgow, UK and ${ }^{2}$ Department of Paediatric \\ Gastroenterology Hepatology and Nutrition, Yorkhill Hospital, Glasgow, UK
}

Crohn's disease (CD) is an uncommon chronic gastrointestinal disorder. The endogenous gut microbiota is believed to be important in its aetiology ${ }^{(1)}$. Exclusive enteral nutrition $(\mathrm{EEN})$ is the mainstream treatment for children with active CD with comparable efficacy to steroids ${ }^{(2)}$. The mechanisms of action remain unknown. Modulation of the dominant gut microbiota metabolic activity has been suggested as a mechanism but has not been addressed by a study yet. The aim of the present study was to investigate the effect of EEN on faecal biomarkers of bacterial metabolic activity in children with active CD.

Fresh stool samples were collected from sixteen children (nine boys) with active CD at four different time points over their 8 weeks of treatment with EEN (Modulen $(\mathrm{B}$; N Nestle, Croydon, Surrey, UK). Faecal biomarkers were measured at different time points (Table) and the clinical disease activity was assessed at baseline and at the end of treatment with a validated paediatric CD activity index (PCDAI).

Seven children achieved clinical remission $(\mathrm{PCDAI} \leq 10)$ and nine had still-active disease (PCDAI $>10$ ). In those children that achieved clinical remission, significant changes in faecal biomarkers were observed (Table). Colonic pH increased to the alkaline range, whereas butyrate production and proportion decreased significantly from baseline. A fivefold increase in the production of total sulphide, but not free sulphide, was observed over the treatment period. D-Lactate but not its L-isomer decreased significantly from baseline. None of these changes were observed for the children with active disease activity (PCDAI >10) at the end their treatment.

\begin{tabular}{|c|c|c|c|c|c|c|c|c|}
\hline \multirow[t]{2}{*}{ Subjects $(n 7)$ at... } & \multicolumn{2}{|c|}{ Baseline } & \multicolumn{2}{|c|}{$15 \mathrm{~d}$} & \multicolumn{2}{|c|}{$30 \mathrm{~d}$} & \multicolumn{2}{|c|}{$60 \mathrm{~d}$} \\
\hline & Mean & SD & Mean & SD & Mean & $\mathrm{SD}$ & Mean & $\mathrm{SD}$ \\
\hline$\overline{\mathrm{pH}}$ & 6.9 & 0.2 & $7.8 * *$ & 0.5 & $7.9 * *$ & 0.3 & $8 * *$ & 0.2 \\
\hline $\mathrm{NH}_{3}(\mathrm{mg} / \mathrm{l})$ & 1596 & 1246 & 756 & 482 & 730 & 324 & 1194 & 1622 \\
\hline Free sulfide $(\mu \mathrm{mol} / \mathrm{g})$ & 0.13 & 0.1 & 0.14 & 0.1 & 0.19 & 0.1 & 0.21 & 0.1 \\
\hline Total sulfide $(\mu \mathrm{mol} / \mathrm{g})$ & 0.6 & 0.3 & $4.7 * *$ & 2.8 & $4.2 * *$ & 2.1 & $3.3^{*}$ & 1.8 \\
\hline Acetate $(\mu \mathrm{mol} / \mathrm{g})$ & 57 & 21 & 61 & 18 & 54 & 15 & 50 & 16 \\
\hline Propionate $(\mu \mathrm{mol} / \mathrm{g})$ & 9.4 & 5.4 & 11.6 & 7.7 & 9.5 & 5 & 7.9 & 3.5 \\
\hline Butyrate $(\mu \mathrm{mol} / \mathrm{g})$ & 15.4 & 7.4 & 9.1 & 6.3 & $7.8 *$ & 3.4 & $6.7 * *$ & 2.9 \\
\hline$\%$ Acetate & 62 & 14 & 66 & 8 & 67 & 9 & 69 & 7 \\
\hline$\%$ Propionate & 9.4 & 4.3 & 12.3 & 5.6 & 11.4 & 4.8 & 11.5 & 5.6 \\
\hline$\%$ Butyrate & 16.9 & 8.8 & $9.3 *$ & 3.8 & $9.8 *$ & 3.6 & $9.3^{*}$ & 3.5 \\
\hline Total SCFA $(\mu \mathrm{mol} / \mathrm{g})$ & 93 & 28 & 93 & 30 & 81 & 18 & 72 & 18 \\
\hline D-Lactate $(\mathrm{mg} / 100 \mathrm{~g})$ & 10.3 & 6.2 & $4.9^{*}$ & 1.1 & $5.2 *$ & 1.3 & 6 & 2.2 \\
\hline L-Lactate $(\mathrm{mg} / 100 \mathrm{~g})$ & 28.9 & 41 & 8.1 & 2.2 & 8.4 & 2.3 & 8.6 & 3.2 \\
\hline Total lactate $(\mathrm{mg} / 100 \mathrm{~g})$ & 39.4 & 45 & 13.3 & 3.4 & 13.9 & 3 & 14.9 & 5.1 \\
\hline$\%$ D-Lactate & 34.8 & 13.7 & 38 & 5.7 & 38.5 & 8.7 & 41.1 & 9.9 \\
\hline$\%$ L-Lactate & 63.8 & 12.5 & 60.4 & 3.5 & 60.1 & 6.3 & 57.6 & 8.6 \\
\hline
\end{tabular}

Values were significantly different from those at baseline (repeated measures ANOVA and Bonferroni post-hoc test): $* P<0.05, * * P<0.01$.

Treatment with EEN resulted in differences in the changes of bacterial metabolic activity between children that achieved clinical remission and those who did not. The results of the study do not support a prebiotic property for EEN as previously speculated ${ }^{(3)}$. EEN induces an 'unhealthy' colonic milieu despite improvement to the disease activity. The lack of indigestible carbohydrates from the nutritional supplement could explain these findings.

1. Sartor RB (2008) Gastroenterology 134, 577-594.

2. Heuschkel RB, Menache CC, Megerian JT \& Baird AE (2000) J Pediatr Gastroenterol Hepatol Nutr 31, 8-15.

3. Lionetti P, Callegari ML, Ferrari S, Cavicchi MC, Pozzi E, de Martino M \& Morelli L (2005) J Parenter Enteral Nutr 29, Suppl., S173-S175. 